\title{
PARAMETRIC SYSTEM IDENTIFICATION APPLIED TO THE BOW SHOCK WAVE SHAPE
}

\author{
G. N. Oliveira ${ }^{a}$, \\ M. G. Silva ${ }^{b}$, \\ and L. R. Carroccic \\ ${ }^{\mathrm{a} D o c t o r}$ in Mechanical Engineering, \\ UNESP - Universidade Estadual Paulista \\ “Júlio de Mesquita Filho”, Faculdade de \\ Engenharia, Campus de Guaratinguetá, \\ Departamento de Energia, Av. Dr. Ariberto \\ Pereira da Cunha 333, CEP: 12510-410, \\ Guaratinguetá, SP, Brasil, \\ g.niederauer@hotmail.com

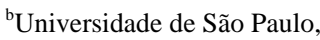 \\ Departamento de Engenharia Mecânica \\ Centro Politécnico, Bairro Jardim das \\ Américas, CP. 19011, Lorena, SP, Brasil, \\ mauriciomgs@iae.cta.br \\ ${ }^{\mathrm{C}}$ UNESP - Universidade Estadual Paulista, \\ “Júlio de Mesquita Filho”, Faculdade de \\ Engenharia, Campus de Guaratinguetá , \\ Departamento de Energia, Av. Dr. Ariberto \\ Pereira da Cunha 333, CEP: 12510-410, \\ Guaratinguetá, SP, Brasil, \\ carrocci@feg.unesp.br \\ Received: July 18, 2012 \\ Revised: May 08, 2013 \\ Accepted: June 10, 2013

\section{ABSTRACT} \\ In this paper it is addressed the prediction of form and location of detached \\ shock waves ahead of two-dimensional and axially symmetric bodies at an \\ zero angle of attack. As shown in Figs. 5 and 6, results show a very good \\ agreement with experimental data. In this context an approximate method, \\ based on a simplified form of the continuity relation, is developed to predict \\ the location of detached shock waves ahead of two-dimensional and axially \\ symmetric bodies. In order to reduce the problem to an equivalent one- \\ dimensional form, it is assumed that: (1) The form of the shock between its \\ foremost point and its sonic point is adequately represented by a hyperbola \\ asymptotic to tile free-stream Mach lines; and (2) the sonic line between the \\ shock and the body is straight and inclined at a constant angle. Although the \\ new methodology has some points of contact with earlier methodologies, \\ the novelty here is that it is used Missile Datcom code as an aid to find out \\ sonic point on body and also it is adopted Parametric System Identification \\ (PSI) in the determination of bow shock shape which uses the Matlab ${ }^{\circledR}$ \\ optimizer fmincon function and an active set strategy to minimize an error \\ in a rms sense subject to simple constraint placed on the parameters by the \\ user. The optimizer function calls a user written function which calculates \\ the shape of the shock wave using the current parameters supplied by \\ optimizer. Also for the shock distance L, the methodology presented here \\ allows to select the value of the mentioned constant angle consistently \\ based either in aerodynamics literature or through physical considerations. \\ As the $\mathrm{L}$ value is previously known from measurements or aerodynamics \\ literature, it was used an optimizer to minimize the error between predicted \\ and known result varying a parameter which absorbs all inconsistencies that \\ arise when it is used the basic Moeckel's model considered here. Once the \\ principal characteristics of the shock wave are calculated, an error value is \\ returned to optimizer function based on the differences between predicted \\ and known results. \\ Keywords: compressible fluid flow, shock wave, shape and position shock

\section{NOMENCLATURE} \\ $A_{S} \quad$ "sonic line” flow area, $\mathrm{m}^{2}$ \\ $A_{0} \quad$ free-stream flow area, $\mathrm{m}^{2}$ \\ $C d_{0}$ discharge-coefficient for area, $A_{0}$ \\ $C d_{S}$ discharge-coefficient for area, $A_{S}$ \\ $\mathrm{k} \quad$ parameter for the PSI approach \\ $L=x_{S B}-x_{0}$ (see Figure 1$)$, $\mathrm{m}$ \\ $M_{0} \quad$ free stream Mach number \\ $M_{S B}$ Mach number on any point of body surface \\ $\dot{m} \quad$ mass flow rate, $\frac{\mathrm{kg}}{\mathrm{s}}$ \\ $P_{0}$ stagnation pressure before shock wave, $\mathrm{N} / \mathrm{m}^{2}$ \\ $P_{S} \quad$ stagnation pressure at sonic point on shock \\ wave, $\mathrm{N} / \mathrm{m}^{2}$ \\ $P_{S}^{A V E R A G E}$ average stagnation pressure on sonic line, \\ $\mathrm{N} / \mathrm{m}^{2}$ \\ $T_{0}$ free stream stagnation temperature, $\mathrm{K}$ \\ $T_{S}$ stagnation temperature at sonic line, $\mathrm{K}$ \\ $x$ abscissa of coordinate system \\ $x_{0}$ abscissa of the shock wave foremost point \\ (see Figure 1) \\ $x_{S}$ abscissa of shock wave sonic point \\ $x_{S B}$ abscissa of body sonic point \\ $y$ ordinate of coordinate system \\ $y_{S}$ ordinate of sonic point on shock wave \\ $y_{S B}$ ordinate of sonic point on body \\ $L_{\text {obs }}$ observed/measured shock distance
}

$B=\frac{A_{S}}{A_{0}}$ 
$L_{\text {pred }}$ predicted shock distance

SSE Sum of Squares due to Error

$\mathrm{R} \cdot \mathrm{sq}$ Ratio of the sum of squares of the regression

A.R.sq Adjusted R.sq

MSE Mean Square Error

RMSE Root Mean Square Error

NSME No Significant Measurement Errors

RE Random Errors

L-15\% L measurement error of - 15\%

$\mathrm{L}+10 \% \mathrm{~L}$ measurement error of $+10 \%$

\section{Greek symbols}

$\beta=\sqrt{M_{0}^{2}-1}=\cot \left[\arcsin \left(\frac{1}{M_{0}}\right)\right]$

$\gamma \quad$ ratio of specific heats (1.4 for air)

$\varphi_{S} \quad$ angle of detached shock at its sonic point, degrees

$\varepsilon \quad$ accepted numerical tolerance

$\eta \quad$ angle between sonic line and normal to free stream direction

$\theta_{S} \quad$ streamline inclination at shock wave sonic point, degrees

$\theta_{S B} \quad$ streamline inclination at body sonic point, degrees

\section{Subscripts}

0 free-stream conditions for Mach number and is related to abscissa of the shock wave foremost point

$\mathrm{S}$ sonic point in shock wave

SB sonic point on body nose

\section{INTRODUCTION}

In recent years arose the interest in the problem of predicting the form and location of detached shock waves. This interest has been stimulated by the necessity for blunt noses and leading edges on configurations designed for hypersonic flight. The ability to predict the form and location of the detached shock is of primary importance in analysis of aerodynamic heating. Knowledge of form and location also has a more elementary use in that it often influences the choice of maximum model size of a given wind tunnel (Pope and Goin, 1965). Numerous experimental and theoretical studies have been devoted to the determination of form and location as well as the important factors influencing form and location (Billig, 1967). If it is considered the theoretical solutions, the solvable fluid flow problems are in general divided into two distinct classes, which are, those in which the field of flow is completely subsonic and those in which the flow is supersonic, each regime having its special methods of solution and approximation. The solution of any fluid flow problem in that region between the critical Mach number and the shock detachment Mach number (transonic region) is a difficulty due in large measure to the combination of mixed subsonic and supersonic flows together with pronounced viscosity or boundary layer effects. In these cases, methods based on Computational Fluid Dynamics (CFD) may be used. With respect to the experimental methods, a number of prediction methods have been shown severely restricted in application (Love, 1957).

It is characteristic of supersonic flight that preceding each body or attached to its nose is a shock wave. Here a differentiation should be made between pointed and blunt-nosed bodies. In the case of blunt bodies, the bow wave always remains detached similar to that shown in Fig. 1. However, for any given sharply pointed body, there is a Mach number below which the shock wave is detached but above which it is attached in the characteristic fashion of a Mach wave, as shown in Boyer (2001). For pointed bodies this Mach number is the detachment Mach number, and, as noted before, represents the upper limit of the transonic region. For blunt nose bodies, on the other hand, there is no upper limit defined. Many of the studies have been centered upon particular important details of the problem, and, therefore, have been locally restricted in scope, for example, studies restricted to hypersonic speeds or to regions in close proximity to the nose. Other studies have been more general in that they present methods for calculating detachment distance and shock shape without restrictions on speed or distance from the body. These methods usually involve laborious iterative procedures since the methodology is based in CFD (Azevedo et al., 1997).

An interesting analytical work is due to Moeckel (1949). In this work an approximate method, based on a simplified form of the continuity relation, is developed to predict the location of detached shock waves ahead of two dimensional and axially symmetric bodies. In order to reduce the problem to an equivalent one dimensional form, it was assumed that the form of the shock between its foremost point and its sonic point is adequately represented by an hyperbola asymptotic to the free stream Mach lines, and the sonic line between the shock and the body is straight and inclined at an angle that depends only on the free stream Mach number. With these assumptions, the location of the shock relative to the body sonic point is independent of the form of the nose or leading edge ahead of the sonic point and becomes a single valued function of the Mach number.

The present research work was undertaken to obtain some rapid information about the geometrical characteristics of detached shock waves. The ultimate objective is to develop an engineering method based on the analytical, experimental and Parametric System Identification (PSI) methods for predicting 
shock shape and detachment distance. It is important to say that this method uses the mathematical formulation data from commercial engineering software, such as Missile Datcom ${ }^{\circledR}$ (Blake, 1998) and MISL3 ${ }^{\circledR}$ (Lesieutre, 2010). In this context, it is possible to include the influence of nose geometry of the vehicle in the determination of detached shock characteristics.

\section{MATHEMATICAL FORMULATION}

This topic presents the mathematical formulation adopted to estimate the distance between the shock wave and the body and the shape of the shock wave in a steady supersonic flow along the body at zero degree of angle of attack. The flow field to be considered is shown in Fig. 1. The methodology is based on the Moeckel (1949), research. Essentially, the mathematical formulation comprises three models, which is: shock wave geometry, shock wave distance from the body and continuity principle.

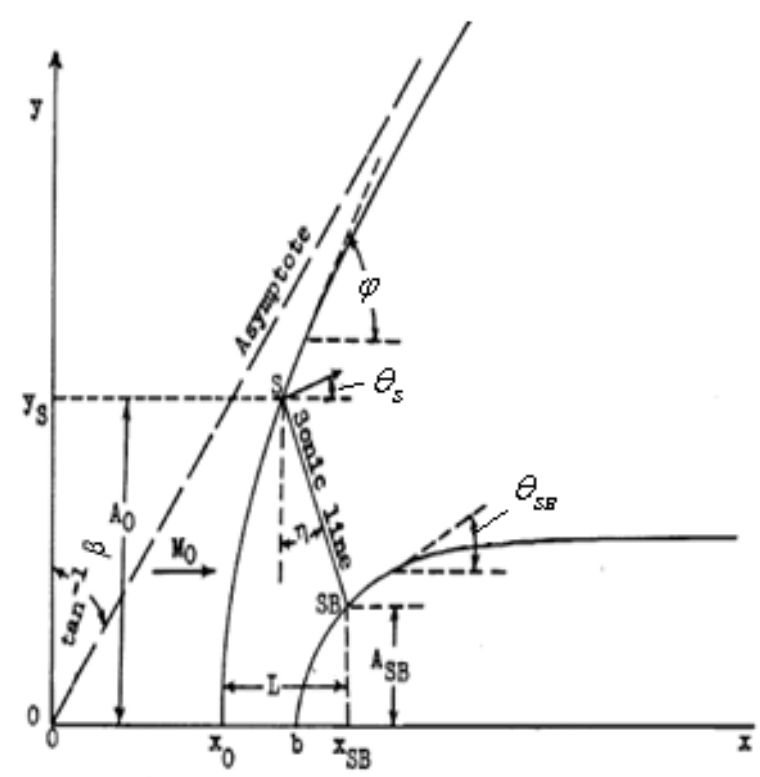

Figure 1. Geometry of the problem (Moeckel, 1949)

\section{Hypothesis}

i. The curved shock is represented by an hyperbola;

ii. The eta angle, $\eta$ (Fig. 1), is a function of flow line deflection at the sonic point of shock wave and flow line deflection at sonic point on the body;

iii. It is adopted the analogy between onedimensional channel flow and flow with detached shock waves. The sonic line is assumed to be normal to the average flow direction in its vicinity;

iv. The influence of nose of the body in the shock wave shape and the distance between the shock wave and the body is obtained from Mach distribution (from commercial software) along the body surface.

\section{Shock Wave Geometry}

The most typical characteristics of detached shock waves are that (Boyer, 2001; Love, 1957; Moeckel, 1949):

i. They are normal to the free stream at their foremost point; and

ii. They are asymptotic to the free-stream Mach lines at large distances from their foremost point.

A simple curve that has these characteristics is a hyperbola, represented by the following equation:

$$
y=\frac{\sqrt{x^{2}-x_{0}^{2}}}{\beta}
$$

The meanings of symbols were already explained in the nomenclature.

With this hypothesis, the angle between the stream direction and the tangent to the shock at any point is given after deriving (1) with respect to $\mathrm{x}$ and finally eliminating $x$. After that the result is:

$$
\frac{d y}{d x}=\tan \varphi=\frac{\sqrt{\beta^{2} y^{2}+x_{0}^{2}}}{\beta^{2} y}
$$

The location of the sonic point at the shock curve $\left(x_{S}, y_{S}\right)$ is given by:

$$
\begin{gathered}
x_{S}=\frac{\beta x_{0}}{\sqrt{\beta^{2}-\cot ^{2} \varphi_{S}}} \\
y_{S}=\frac{x_{0} \cot \varphi_{S}}{\beta \sqrt{\beta^{2}-\cot ^{2} \varphi_{S}}}
\end{gathered}
$$

If the $y$-coordinate of the body sonic point $\left(y_{S B}\right)$ is used as the reference dimension, then the form of the shock wave can be modeled by:

$$
\frac{y}{y_{S B}}=\frac{1}{\beta} \sqrt{\left(\frac{x}{y_{S B}}\right)^{2}-\left(\frac{x_{0}}{y_{S B}}\right)^{2}}
$$

From the definition of $y_{S}$ it is possible to conclude:

$$
\frac{x_{0}}{y_{S B}}=\beta \frac{y_{S}}{y_{S B}} \sqrt{\beta^{2} \tan ^{2} \varphi_{S}-1}
$$


In this context, the dimensionless location of the shock sonic point is given by:

$$
\frac{x_{S}}{y_{S B}}=\frac{\beta \frac{x_{0}}{y_{S B}}}{\sqrt{\beta^{2}-\cot ^{2} \varphi_{S}}}
$$

\section{Shock Wave Distance}

Figure 1 illustrates the geometry of the problem considered. It is possible to conclude that the distance from the foremost point of the shock $\left(x_{0}\right)$ to the $\mathrm{x}$ coordinate of the body sonic point $\left(x_{S B}\right)$ is related to the distance between the shock and the body (Moeckel, 1949) in accordance with the expression:

$$
\frac{L}{y_{S B}}=\frac{x_{S B}}{y_{S B}}-\frac{x_{0}}{y_{S B}}
$$

But:

$$
\frac{x_{S B}}{y_{S B}}=\frac{x_{S}}{y_{S B}}+\left(\frac{y_{S}}{y_{S B}}-1\right) \tan \eta
$$

Thus:

$$
\frac{L}{y_{S B}}=\frac{y_{S}}{y_{S B}}(C+\tan \eta)-\tan \eta
$$

Where:

$$
C=\beta\left(\beta \tan \varphi_{S}-\sqrt{\beta^{2} \tan ^{2} \varphi_{S}-1}\right)
$$

In MOECKEL’s model:

$$
\eta=\frac{\theta_{S}+\theta_{S B}}{2}
$$

In which the variable $\theta_{S}$ represent the flow line deflection at the sonic point of shock wave and $\theta_{S B}$ is the flow line deflection at sonic point at the body. In this paper $\eta$ is selected from available data from experiments or aerodynamics literature and physical reasoning.

\section{Continuity Principle}

In order to estimate the quantity $\frac{y_{S}}{y_{S B}}$ the continuity relation, for axially symmetric bodies, is applied to the fluid that passes through free-stream area:

$$
A_{0}=\frac{\pi}{4} y_{S}^{2}
$$

and area corresponding to sonic line:

$$
A_{S}=\frac{\pi}{4}\left(\frac{y_{S}^{2}-y_{S B}^{2}}{\cos \eta}\right)
$$

The integral form of this relation is useless for the present method, inasmuch as the distribution of the flow variables along the sonic line is unknown. The distribution of stagnation pressure immediately behind the shock, however, is known. Continuity principle is defined by the analogy between onedimensional channel flow and flow with detached shock waves, the sonic line is assumed to be normal to the average flow direction in its vicinity. From this hypothesis:

$$
\begin{aligned}
& \dot{m}=P_{0} A_{0} C d_{0}\left(\gamma, T_{0}, M_{0}\right)= \\
& P_{S} A_{S} C d_{S}\left(\gamma, T_{S}=T_{0}, M_{S}=1\right)
\end{aligned}
$$

Since the stagnation pressure remains constant along each streamline behind the shock, it is adopted a hypothesis which state that there is a value for “average” stagnation pressure $\left(P_{S}^{\text {Average }}\right)$ will remain unchanged between the shock wave and the sonic line. Because the total temperature is constant, the simplified continuity equation may be written as:

$$
\frac{A_{S}}{A_{0}}=M_{0} \frac{P_{0}}{P_{S}^{\text {Average }}}\left[\frac{2+(\gamma-1) M_{0}^{2}}{\gamma+1}\right]^{-\frac{\gamma+1}{2(\gamma-1)}}
$$

Using the area relation in the Fig. 1, it is possible to write:

$$
\frac{y_{S}}{y_{S B}}=(1-B \cos \eta)^{-\frac{1}{2}}
$$

Where:

$$
B=M_{0} \frac{P_{0}}{P_{S}^{\text {Average }}}\left[\frac{2+(\gamma-1) M_{0}^{2}}{\gamma+1}\right]^{-\frac{\gamma+1}{2(\gamma-1)}}
$$

In this context, the distance $L$ of shock can be expressed as:

$$
\frac{L}{y_{S B}}=(1-B \cos \eta)^{-\frac{1}{2}}(C+\tan \eta)-\tan \eta
$$

\section{NUMERICAL ALGORITHM}

The shock wave divides the field into two parts. Everywhere upstream of the shock wave, the flow is uniform and the total head or stagnation pressure is constant. Downstream of the shock wave, the flow varies throughout the field and each stream line has a different stagnation pressure. This variation in stagnation pressure is due to the entropy change 
through the shock wave. The entropy change is primarily a function of the free stream Mach number and the angle of deflection of flow line $\left(\theta_{S}\right)$. The methodology developed by Moeckel (1949), uses an appropriate average value for stagnation pressure. This average value is assumed to be a quantity that exists along the streamline which represents the mass centroid of the fluid passing the sonic line. This centroid streamline enters the shock wave at $y_{C}=\frac{y_{S}}{2}$ for plane flow and at $y_{C}=\frac{2 y_{S}}{3}$ for axially symmetric flow. In this paper, it is developed a methodology which does not uses this approach. It is used a numerical algorithm from which it is possible to estimate the shock wave shape and location. The following paragraphs describe the numerical algorithm:

i. Input data: Free stream condition $\left(M_{0}, P_{0}, T_{0}\right)$, geometry of the body $\left(y_{S B}=y_{S B}\left(x_{S B}\right)\right)$, Mach distribution on the body $\left(M_{S B}=M_{S B}\left(x_{S B}, y_{S B}\right)\right)$, a cloud of measured pair of data points of bow shock shape, and a measured distance L;

ii. Determine $y_{S B}=y_{S B}\left(M_{S B}=1\right)$ : location on the body in which Mach number is equal one;

iii. Determine $x_{0}$ and $\beta$ via a dedicated Matlab program specifically written for problems of this type which considers $x_{0}$ and $\beta$ as parameters to be determined minimizing an error in a rms sense in a Grey Box type model of a Parametric System Identification algorithm using an optimizer. In this way, equation (1) is numerically completely defined.

iv. Determine the wave shock angle $\left(\varphi_{S}\right)$ at the sonic point at the shock wave:

$$
M_{A f t e r}^{2}=\frac{1+\frac{\gamma-1}{2} M_{0}^{2}}{\gamma M_{0}^{2} \sin ^{2} \varphi_{S}-\frac{\gamma-1}{2}}+\frac{M_{0}^{2} \cos ^{2} \varphi_{S}}{1+\frac{\gamma-1}{2} M_{0}^{2} \sin ^{2} \varphi_{S}}=1
$$

v. Determine $C$ using equation (11).

vi. Use equation (19) do determine numerically distance $\mathrm{L}$.

As the basic model used here is approximate and in order to get predicted values very close to real cases it is necessary to use in equation (19) a parameter $k$ in the following way:

$$
\begin{aligned}
L_{\text {pred }}=y_{S B} \cdot\{1-B \cdot[k \cdot \cos (\eta)]\} \cdot{ }^{-0.5} k \cdot[C+\tan (\eta)] \\
-y_{S B} \cdot[k \cdot \tan (\eta)]
\end{aligned}
$$

In this equation, $\eta$ can be selected according available data from experiments or aerodynamic literature. It is very important to note that once $\eta$ is selected, $B$ is also automatically selected from equation (17):

$$
B=\left[1-\left(\frac{y_{S B}}{y_{S}}\right)^{2}\right] \cdot \frac{1}{\cos \eta}
$$

In this equation $y_{S}$ is determined through intersection with the straight line starting from $x_{S B}$ and $y_{S B}$, inclination $(\eta+90)$ degrees with the previously numerically defined bow shock shape hyperbola, equation (1). The expected value of $B$ should lies between 0 and 1 .

So $L_{\text {pred }}$ becomes a single valued function from $k$. The value of $k$ is determined minimizing equation:

$$
\varepsilon=\text { error }=\frac{\left(L_{\text {pred }}-L_{\text {obs }}\right)}{L_{\text {pred }}}
$$

in which $L_{o b s}$ is the observed/experimental value for $L$. This minimization is done via an optimizer.

Also the idea shown in this text should be repeated for a wide range of Mach numbers, for the same nose, in order to get a function $\mathrm{k}=\mathrm{k}$ (Mach) which will allow to readily determine $\mathrm{L}$ from each Mach number.

vii. Determine distance $\Delta$ between the tip of body nose and foremost point $\mathrm{x} 0$ :

The commercial engineering software used (Missile Datcom) places the origin of coordinates system at the tip of body nose. So the originally determined $x_{S B}$ and $y_{S B}$ values are with respect to the tip of nose body. Thus:

$$
L=\Delta+x_{S B} \quad \therefore \Delta=L-x_{S B}
$$

viii. Show figure informing simultaneously body nose shape, bow shock wave shape, body sonic point coordinates, shock sonic point coordinates and distance between the tip of body nose and foremost point ${ }^{x_{0}}$ :

With $\Delta$, originally determined body nose coordinates and calculated bow shock wave points coordinates this is easily done using the Matlab programs already mentioned.

\section{PARAMETRIC SYSTEM IDENTIFICATION}

It is very important to remember here that Moeckel's bow shock model is a simplified model. Thus it is worthwhile to use some parameters to adjust the original model equations in order to get realistic values. In this paper this is done to equation for $L_{\text {pred }}$, which was multiplied by a parameter $k$ to be determined with the methodology already 
described.

The purpose is to match calculated shock wave distance with known aerodynamic literature data, or experimental data, in order to validate the numerical approach. Essentially, the parametric system identification (PSI) consists to include an optimizer that could adjust the unknown parameters or variables until a best-fit approximation in an rms sense to experimental data is obtained. Parameter identification has several advantages over the other modeling choice as, for example, computational fluid dynamics (CFD). Creating a mathematical model which capture the shock waves characteristics using CFD is feasible, however, extremely expensive. The objective of this paper is to develop an engineering method which provides fast and low cost results. PSI ignores the complexities of the flow field (shock waves, boundary layer, and so on) and focuses on the end results. This makes PSI not only simpler, but the information that comes out of the PSI model can easily be used in improving the design. Another interesting aspect of this methodology is that PSI also handles nonlinear conditions such as heat transfer, which can be implemented in a future work.

The PSI procedure adopted in this work for determining $x_{0}$ and $\beta$ follows the reasoning line: firstly, physical and geometric data is read in from a data file. The optimizer function then calls a user written function which calculates the shape of the shock wave starting with the guessed parameters values $\beta$ and $x_{0}$ supplied to the optimizer. From these initial information the optimizer, at each iteration step, adjusts the unknown parameters $x_{0}$ and $\beta$ minimizing an error in an rms sense to experimental data until a best-fit approximation is obtained. The optimizer used was fmincon function from Matlab ${ }^{\circledR}$.

To calculate the optimized value of $k$ that enables to get a realistic shock distance $L_{\text {pred }}$, it is used the numerical algorithm aforementioned. For this the initial value of $k$ is guessed and therefore, it is necessary to update it. In this numerical algorithm the optimizer function calls a user written function which calculates the optimized value of $k$ starting from the corresponding initially guessed value. During this calculation, once $k$ is calculated, an error value is returned to optimizer function based on the differences between predicted $L_{\text {pred }}$ and observed/experimental $L_{o b s}$ shock distance. The optimizer then adjusts the unknown parameter $k$ and the process repeats until certain convergence criterion is met. For this, it is also used the optimizer fmincon function from Matlab ${ }^{\circledR}$, to minimize the error between observed/experimental $L_{o b s}$ and predicted/calculated $L_{\text {pred }}$. It was observed that for low Mach number as 1.5 the value of $B$ was unrealistic $(B \approx 1)$. This can be clearly understood when the equation (22) for $B$ is examined. There, as the Mach number tends to $1, y_{S}$ grows very much and $\eta$ tends simultaneously to zero. This happens because the reference model used here is approximated.

\section{RESULTS}

In this section it is presented some results when the numerical algorithm is implemented via Matlab ${ }^{\circledR}$. In this sense two different types of body nose were analyzed: Ogive and Hemispheric (H-spheric) nose. Firstly it is presented the results considering no significant errors in measurements. It is important to emphasize that the methodology was developed to treat flow field with zero degree of angle of attack. Secondly it is analyzed what happens when there are significant errors on the data points measurements. Thirdly, it is analyzed cases in which there are uncertainties about the shock location.

\section{Geometrical configurations}

The geometry of the Ogive nose, drawn using Missile Datcom ${ }^{\circledR}$, is shown in the Fig. 2, and the geometry of the Hemispherical nose is shown in the next Fig. 3. It is important to emphasize that the choice of these geometries for nose shape was based on the practical application in the aerodynamic design of aeronautical vehicles.

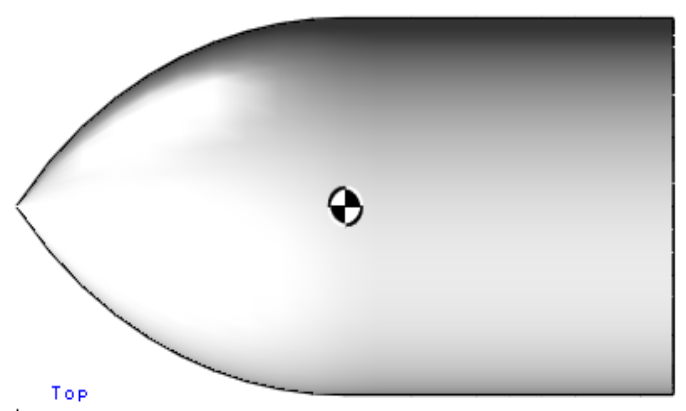

Figure 2. Ogive body nose.

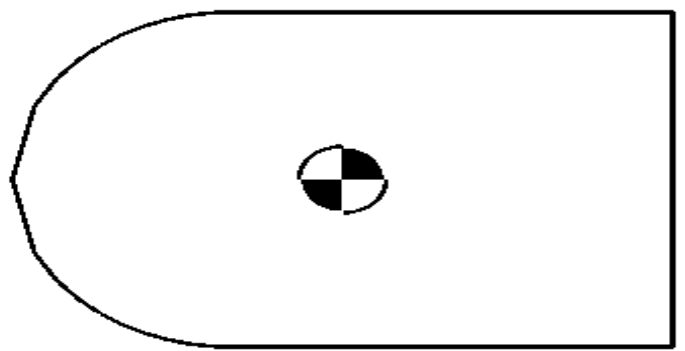

Figure 3. Hemispherical body nose.

\section{Numerical Results}

Table 1 shows the conditions used in the numerical simulations. For each nose and respective Mach number, Missile Datcom ${ }^{\circledR}$ gave all pertinent 
flight data including Mach number information closely to the body wall. These data were implemented in a dedicated Matlab ${ }^{\circledR}$ program and the corresponding bow shock wave shape was obtained.

Table 1. Simulation conditions.

\begin{tabular}{|c|c|}
\hline Mach number & $1.5,3.0$ \\
\hline Diameter (D) & 1 length unit \\
\hline Nose length & 2 D \\
\hline
\end{tabular}

Figure 4 shows the Mach distribution on the wall of ogive for various simulation conditions. It is important to note that in all cases it was found Mach 1 (one) along a circumferential line on the wall. The numerical algorithm fails if this constriction is not met. For no significant measurements errors, Figs. 5 and 6, shows the comparative analysis between the theoretical and experimental results for the shock shape and distance of shock. In each of these figures there is illustrated the sonic point at the body and shock wave, the experimental results and the shock shape obtained from numerical algorithm above mentioned. The calculated parameters for Fig. 5 are $\beta$ $=1.118$ and $x_{0}=5.223$ and for Fig. 6 are $\beta=2.228$ and $x_{0}=5.783$. For each Fig. 5 and 6 it was done the corresponding Goodness of Fit analysis. The corresponding values are in Tab. 2 - Goodness of Fit Data. As can be seen from Figs. 5 and 6 and Tab. 2, there is a very good agreement with theoretical results and experimental data.

For significant errors on the data points measurements, Figs. 7 and 8 show the shape shock results when there are random errors on the bow shock shape data points measurements. In Fig. 7 the absolute value of the average random error is 0.693 and the calculations gave $\beta=1.057$ and $x_{0}=5.930$ instead of $\beta=1.118$ and $x_{0}=5.223$. In Fig. 8 the absolute value of the average random error is 0.076 and the calculations gave $\beta=2.335$ and $x_{0}=4.000$ instead of $\beta=2.228$ and $x_{0}=5.783$. The objective is to evaluate the flexibility of the numerical algorithm in the treatment of errors in the experimental measurements. For each Fig. 7 and 8 it was done also the corresponding Goodness of Fit analysis in Tab. 2. Also theoretical results were obtained based only on experimental data with random error measurements. From Figs. 7 and 8 and Tab. 2, it can be seen that although no agreement with theoretical results and experimental data with random error was achieved a better fitting was achieved for Fig. 8 with a R·sq (R_square) $=0.999$. As an overall observation from Figs. 7 and 8 , it can be concluded that the methodology used here is very sensitive of the accuracy of the shock shape data point measurements.

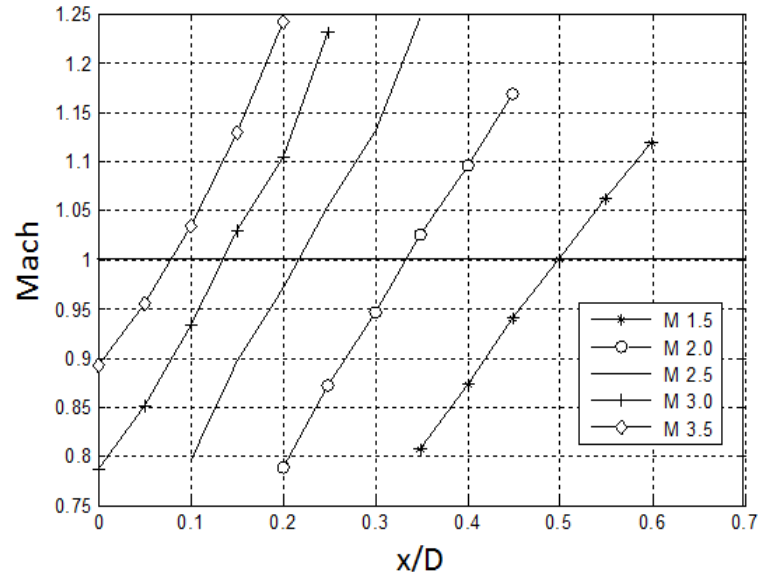

Figure 4. Mach distribution (Ogive nose).

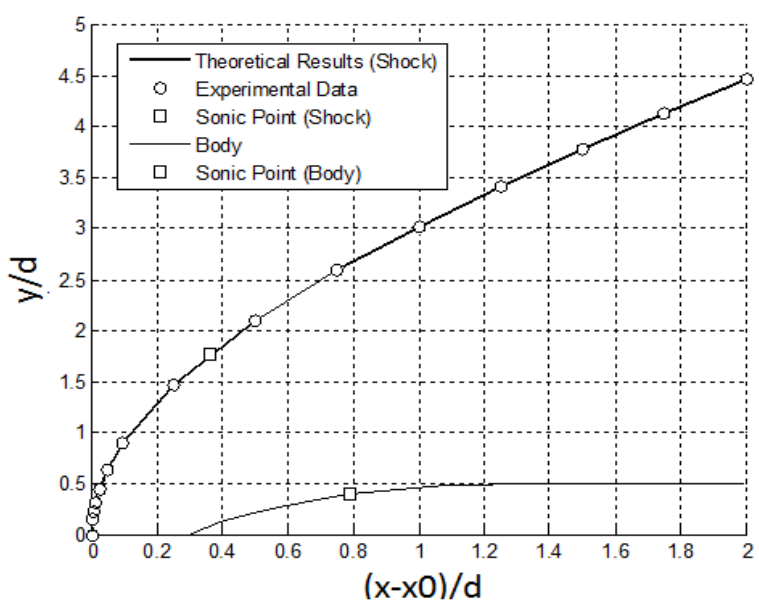

Figure 5. Shock shape - Ogive nose ( Mach=1.5).

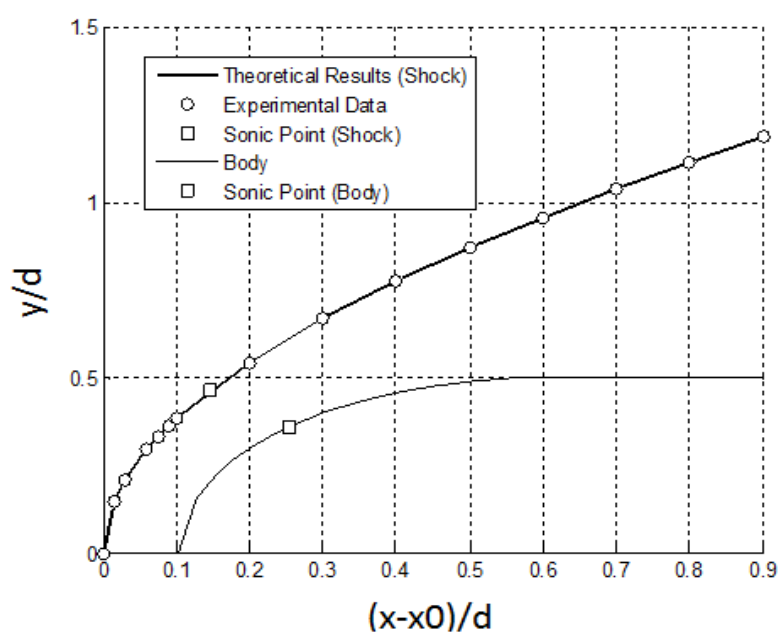

Figure 6.Shock shape - H-spheric. nose ( Mach=3.0). 


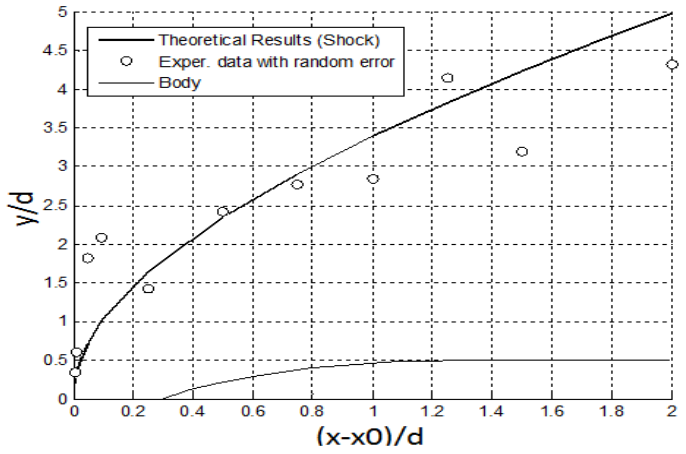

Figure 7. Shock shape - Ogive Nose ( Mach=1.5, with RE).

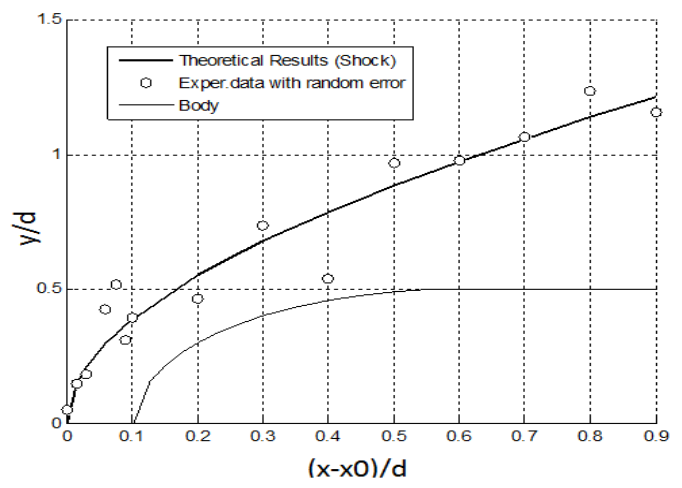

Figure 8. Shock shape - H-spheric. nose ( Mach=3.0, with RE).

Figures 9 and 10 show what happens when there are uncertainties about the shock location $L$. For each figure it was considered two situations: one in which there is an uncertainty up to $-15 \%$ in L length and another in which there is an uncertainty up to + $10 \%$ in $\mathrm{L}$ length. For each figure it was done also the corresponding Goodness of Fit analysis. The corresponding values are in Tab. 2 - Goodness of Fit Data. It can be observed from Figs. 9 and 10 that an error measurement of $10 \%$ keeps the resulting shock shape closer to experimental data when compared with an error measurement of 15\%, as shall be.

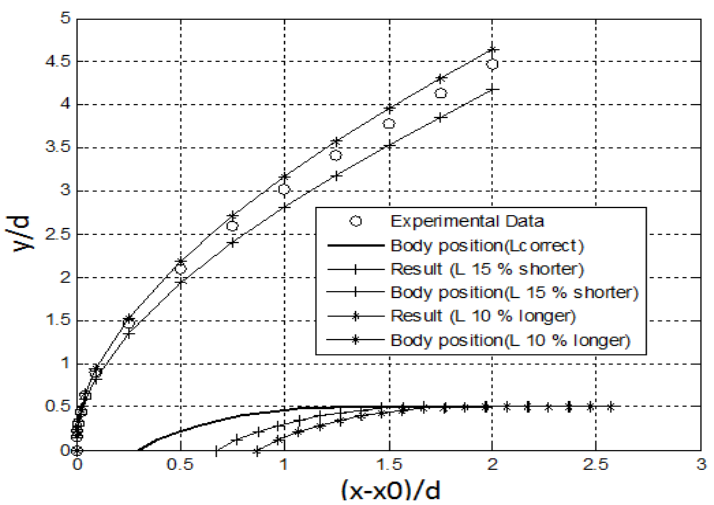

Figure 9. Shock shapes - - Ogive Nose ( Mach=1.5, with L measurement errors).

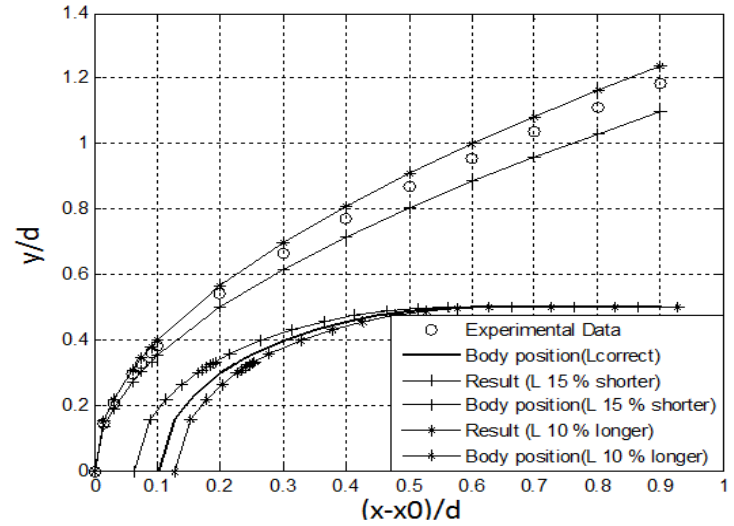

Figure 10. Shock shapes - H-spheric. nose ( Mach=3.0, with L measurement errors).

Table 2. Goodness of fit data.

\begin{tabular}{|c|c|c|c|c|c|}
\hline O GIVE & SSE & R·sq & A·R·sq & MSE & RMSE \\
\hline NSME & 0.000 & 1.000 & 1.000 & 0.000 & 0.000 \\
\hline RE & 1.236 & 0.966 & 0.960 & 0.095 & 0.308 \\
\hline L $+15 \%$ & 0.410 & 0.989 & 0.988 & 0.029 & 0.171 \\
\hline L - $10 \%$ & 0.163 & 0.995 & 0.995 & 0.012 & 0.108 \\
\hline H-SPHERIC & SSE & R.sq & A·R·sq & MSE & RMSE \\
\hline NSME & 0.000 & 1.000 & 1.000 & 0.000 & 0.000 \\
\hline RE & 0.003 & 0.999 & 0.999 & 0.000 & 0.014 \\
\hline L +15\% & 0.040 & 0.981 & 0.979 & 0.003 & 0.053 \\
\hline L - $10 \%$ & 0.016 & 0.992 & 0.992 & 0.001 & 0.033 \\
\hline
\end{tabular}

\section{FINAL COMMENTS AND CONCLUSIONS}

Using simple concepts for predicting the shape and location of detached bow shock waves it was shown how to calculate these data in a reliable fashion as shown by the figures presented in this text. From the results presented in the previous section, it is possible to conclude:

$>$ The engineering method described can be used for the first estimate for shape and shock location;

$>$ The algorithm uses data from commercial software (in this case, Missile Datcom ${ }^{\circledR}$ ). In this context, the algorithm can be linked to this kind of software in order to obtain automatically the first idea of the shape and the distance of the shock from the body;

$>$ The real shock line is not a straight line, but a curved line. So the angle $\eta$ is not constant, as assumed during the calculations, but really varies along the sonic line. Owing to the hypothesis of a straight sonic line, the real flow exit surface is curved and with its numerical value different from the assumed straight line. It was introduced a parameter $k$ in order to reproduce the influence aforementioned;

$>$ Detached wave standoff distance: physically this distance arises due to energy equilibrium between the flow into and flow out in order to accommodate the corresponding temperature, density, velocity, pressure and momentum changes in the flow field owing to the presence of the body in the flow. The complete calculation procedure is also to check 
calculated values against the measured values. When this is done it is noted that some difference arises. These numerical differences mainly occur due to the simplified method of calculation used in this paper. It is suggested here that new improvements in this method of calculation shall be done to improve it more, for instance, using a new relationship for the $\eta$ (eta) angle based on the results from the CFD methods. It can be proved that the shape and location of the shock is very sensitive with the eta angle.

\section{REFERENCES}

Azevedo, J. L. F., Strauss, D., and Ferrari, M. A. S., 1997, Viscous Multiblock Simulations of Axisymmetric Launch Vehicle Flows, AIAA Paper No. 97-2300, Proc. of 15th AIAA Applied Aerodynamics Conference, Atlanta, GA.

Billig, F. S., 1967, Shock-Wave Shapes around Spherical and Cylindrical-Nosed Bodies, J.Spacecraft, Vol. 4, No. 6, pp. 822-823.

Blake, W. B., 1998, Missile Datcom User’s Manual, AFRL-VA-WP-TR-1998-3009.

Boyer, K. M., 2001, An Improved Streamline Curvature Approach for Off-Design Analysis of Transonic Compression Systems, Doctoral Thesis, Virginia Polytechnic Institute and State University.

Lesieutre, D., J., 2010, MISL3 Aerodynamic Analysis for Finned Vehicles with Asymmetrical Bodies, NEAR TR-654.

Love, E. S., 1957, A Reexamination of the Use of Simple Concepts for Predicting the Shape and Location of Detached Shock Waves, NACA TN 4170, Langley Aeronautical Laboratory, Langley Field, Va.

Moeckel, W. E., 1949, Approximate Method for Predicting Form and Location of Detached Shock Waves Ahead of Plane or Axially Symmetric Bodies,

Naca, T. N., 1921, Lewis Flight Propulsion Laboratory, Cleveland, Ohio.

Pope, A., and Goin, K. L., 1965, High Speed Wind Tunnel Testing. New York: John Wiley. 